31:384-387). The criteria proposed for office diagnosis of migraine without aura (common migraine) are as follows (any two of the four features): (1) headache - unilateral; (2) headache - pulsating quality; (3) associated nausea; (4) associated photophobia or phonophobia. If preceded within one hour by visual, sensory, or motor symptoms lasting 5 to 60 minutes the headaches are diagnosed as migraine with aura (classical migraine). Organic disease must be ruled out and the patient should have had several similar attacks in the past. Tension type headache is usually diagnosed by negative criteria and by ruling out migraine, cluster headache and headaches due to organic disease. Cluster headaches are differentiated from migraine by the frequency of attacks (daily) and the associated autonomic symptoms (ipsilateral conjunctival injection, tearing, nasal congestion, ptosis or miosis).

\title{
EPISODIC MYDRIASIS AS MIGRAINE EQUIVALENT
}

A 14-year-old girl who presented with yearly attacks of bilateral internal ophthalmoplegia, nausea and headache since the age of 9 is the subject of a case report from the Department of Child Neurology, University Hospital Nijmegen, The Netherlands. She complained of blurred near vision when reading, associated with photophobia, phonophobia, nausea and aspirinresistant bitemporal headaches. Attacks lasted 1 to 7 days. The pupils were symmetrically dilated during the attack and there was no response to direct or consensual light with complete loss of accommodation. The father and paternal aunt had migraine (van Engelen BGM et al. Bilateral episodic mydriasis as a migraine equivalent in childhood: a case report. Headache June 1991; $\underline{31: 375-377) . ~}$

COMMENT. The authors note that ophthalmoplegic migraine with bilateral mydriasis has not previously been described in children. A single ischemic lesion localized in the central parasympathetic nucleus of Perlia and the Edinger-Westphal nuclei is proposed as the likely mechanism for the bilaterality of the mydriasis. Other causes including drugs, deodorant-antiperspirants, perfumes were excluded.

Unusual clinical manifestations of migraine are reviewed by Ehrenberg BL in Seminars in Neurology June 1991; 11:118-127. Some patients have headaches without aura, some have confusional spells, memory lapses and episodes of speech arrest resembling complex partial seizures. Basilar migraine is manifested by auras of vertigo, dysarthria, tinnitus, decreased hearing, diplopia, disturbances in both visual fields, bilateral sensory loss or weakness, or alterations in consciousness. The syndrome of benign epilepsy of childhood with occipital paroxysms is preceded by hallucinations or visual loss and associated with occipital spike-wave activity on interictal EEG. Episodes with auras or hallucinations lasting 5 to 60 minutes resemble migraine attacks. Numerous studies show a high incidence of EEG abnormalities in large populations of migraine patients (Millichap JG Child's Brain $1978 ; \underline{4}: 95-105)$. A beneficial response to phenytoin in 
this and other studies does not contraindicate a diagnosis of migraine. Migraine-epilepsy syndromes are reviewed by Andermann F. Boston: Butterworths $1987 ; 407-8$. These include epileptic seizures induced by a classic migraine aura and seen more often in children than in adults.

\section{MIGRAINE AND EPILEPSY: INFANTILE ONSET}

The coexistence of classical migraine, visual phenomena and seizures and occipital spike-wave complexes in the EEG are reported in 14 children from the Department of Neurological Sciences, University La Sapienza, Rome, Italy. The age of onset was 3 to 9 years (mean age 5.4 years) and the clinical and EEG follow-up ranged from 4 to 16 years (mean 11.2 years). Visual phenomena were described as bright rings, bands, red-colored disks, in both visual fields or in the visual hemifield opposite the spike-wave localization. Amaurosis followed the appearance of the phosphenes. In some patients the migraine with aura and epilepsy were chronologically related phenomena; migraine might occur immediately before or after seizures; migraine may act as a triggering factor for epilepsy. In others, visual phenomena either preceded migraine with aura or occurred as an isolated event or even preceded epileptic seizures (De Romanis F et al. Migraine and epilepsy with infantile onset and electroencephalographic findings of occipital spike-wave complexes.

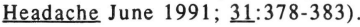

COMMENT. In $50 \%$ of the patients in this study, migraine with aura, with phosphenes and amaurosis as prodromal symptoms, replaces epileptic seizures when they disappear or improve following antiepileptic treatment. Ergot alkaloids or pizotifen were of little or no benefit whereas migraine with aura responded to anti-epileptic treatment. Occipital epilepsy in childhood in this study was not benign and seizures were controlled by anti-epileptic treatment in only 2 subjects.

Migraine and pregnancy is reviewed by Uknis A and Silberstein SD in the same issue of Headache 1991; 31:372-374. A first attack of migraine with unilateral parasthesias and blurred vision occurred in a 27-year-old woman during the first trimester and led to the diagnosis of her pregnancy. Pre-existing migraine usually improves with pregnancy, whereas relapse frequently occurs in the post-partum period. Children born to migraineurs have no increased incidence of birth defects.

\section{SEIZURE DISORDERS}

\section{PROGNOSIS OF NEONATAL SEIZURES}

The neurologic outcome of 40 infants with EEG documented seizures of diverse etiologies was examined retrospectively at the Division of Neurology, The Children's Hospital of Philadelphia, PA. Neurologic followup of 27 survivors performed at a mean age of 31 months (range 5 to 56 